\title{
Prevention and Management of Ovarian Hyperstimulation Syndrome
}

\author{
Sonal Agarwal ${ }^{1}$, Deepika Krishna ${ }^{2}, K^{2}$ amini A Rao ${ }^{3}$
}

\begin{abstract}
Ovarian hyperstimulation syndrome (OHSS) is a potential iatrogenic life-threatening situation. It is difficult to decipher OHSS pathophysiology. ${ }^{1}$ The occurrence is directly proportional to estradiol in blood, follicle number, and human chorionic gonadotropin (hCG) with more chances of happening in polycystic ovarian disease. Complete prevention of OHSS is never possible, but endocrine profile and ultrasonographic follicular monitoring are the mainstay of its prediction. Complications such as hemoconcentration, hypovolemia, and thromboembolism can occur. Withholding hCG, continuation of gonadotropin-releasing hormone analogs, coasting, agonist trigger, intravenous albumin, dopamine agonists, and cryopreservation of embryos are cornerstones of OHSS prevention. ${ }^{2}$ Mild OHSS usually requires no active therapy. Moderate and severe cases have to be treated. Surgery may be needed in ruptured ovarian cysts, torsion, or concomitant ectopic pregnancy. Transvaginal paracentesis is recommended in cases of severe ascites. Inpatient management is typically based on preventing complications such as derangement of kidney and liver functions, thrombosis, and severe respiratory depression. Recent ART treatment protocols behold more patient-friendly mild ovarian stimulation regimes that are individualized depending on patient's ovarian reserve. ${ }^{3}$

Keywords: Electrolyte imbalance, Ovarian hyperstimulation syndrome, Paracentesis, Prevention.

International Journal of Infertility and Fetal Medicine (2019): 10.5005/jp-journals-10016-1193
\end{abstract}

\section{INTRODUCTION}

Controlled ovarian stimulation according to the International Society for Mild Approaches in Assisted Reproduction (ISMAAR) classification is based on producing multiple ovarian follicle $(>-8)$ in an already ovulating women. It is a hallmark in any assisted reproduction cycle in desire to increase number of oocytes for fertilization and development of embryo. Ovarian hyperstimulation syndrome (OHSS) is an iatrogenic complication of supraphysiologic ovarian stimulation. ${ }^{4}$ It is almost exclusively associated with exogenous gonadotropin stimulation and rarely associated with clomiphene citrate stimulation or spontaneous ovulation. ${ }^{5}$ Prevalence of severe Ovarian hyperstimulation syndrome is $1.4 \%$ of all cycles. ${ }^{6}$ Mortality risk is evaluated as around one in approximately four to five lakh cases. OHSS is an amplified reaction to ovarian stimulation pertaining to intravascular protein-rich fluid shift to third space. Clinicians prescribing different protocols for ovarian stimulation should be well versed with their pharmacokinetics and have complete knowledge about selecting protocol for particular patient. Once at risk, clinician should be able to prevent it, treat it, and manage it properly. Aim should be to identify women at risk and apply preventive strategies. Most OHSS is mild and of little clinical concern. Severe OHSS can lead to significant morbidity and mortality. Reported incidence of moderate variety of OHSS is 3-6\%, while severe is $0.1-2 \%$. The mild form occurs in about $20-33 \%$ of IVF cycles. ${ }^{7}$ Finnish registry in 2005 concluded incidence of severe OHSS of $1.4 \%$ per cycle.

Prevention of OHSS sets its first milestone by tailoring stimulation protocol of a particular patient according to their own risk profile, i.e., individualized controlled ovarian stimulation. Benefits and risks associated with each strategy are different for each cohort of people due to heterogeneous profiles of patients. Selecting best preventive approach is challenging.Way of completely preventing it is still a dilemma. There are some avenues and stones that can be thrown to reduce its incidence and severity.
${ }^{1-3}$ Department of Reproductive Medicine, Milann Fertility Centre, Bengaluru, Karnataka, India

Corresponding Author: Sonal Agarwal, Department of Reproductive Medicine, Milann Fertility Centre, Bengaluru, Karnataka, India, Phone: +918094598845, e-mail: sonaljaipur28@gmail.com

How to cite this article: Agarwal S, Krishna D, Rao KA. Prevention and Management of Ovarian Hyperstimulation Syndrome. Int J Infertil Fetal Med 2019;10(3):46-51.

Source of support: Nil

Conflict of interest: None

\section{Pathophysiology}

Ovarian hyperstimulation syndrome is a systemic complication arising due to activation of vasoactive substances that are let out of hyperstimulated ovaries. Capillary permeability expels fluid rich in protein into third space resulting in hemoconcentration. Hypercoagulability and reduced end-organ perfusion emanates. ${ }^{8}$ Human chorionic gonadotropin (hCG) is considered to be the critical element in mediating this syndrome. ${ }^{9}$ Role of hCG is found in two different but significant clinical presentations of OHSS: Early and late. ${ }^{10}$

"Early" OHSS happens within 1 week of hCG trigger representing exogenous hCG effect on ovaries hyperstimulated by gonadotropins. "Late" OHSS generally occurs approximately 10 days after use of hCG trigger. It is due to endogenous hCG production by trophoblast and not the exogenous hCG administration. hCG has a very important role in pathophysiology of OHSS with VEGF-A as the primary mediator interacting with VEGF receptor-2 causing increase in vascular permeability (Fig. 1). VEGF-A concentration is elevated in women at risk of OHSS. ${ }^{11}$ Human chorionic gonadotropin stimulates granulosa-lutein cells leading to the increased production of growth factor mRNA which 


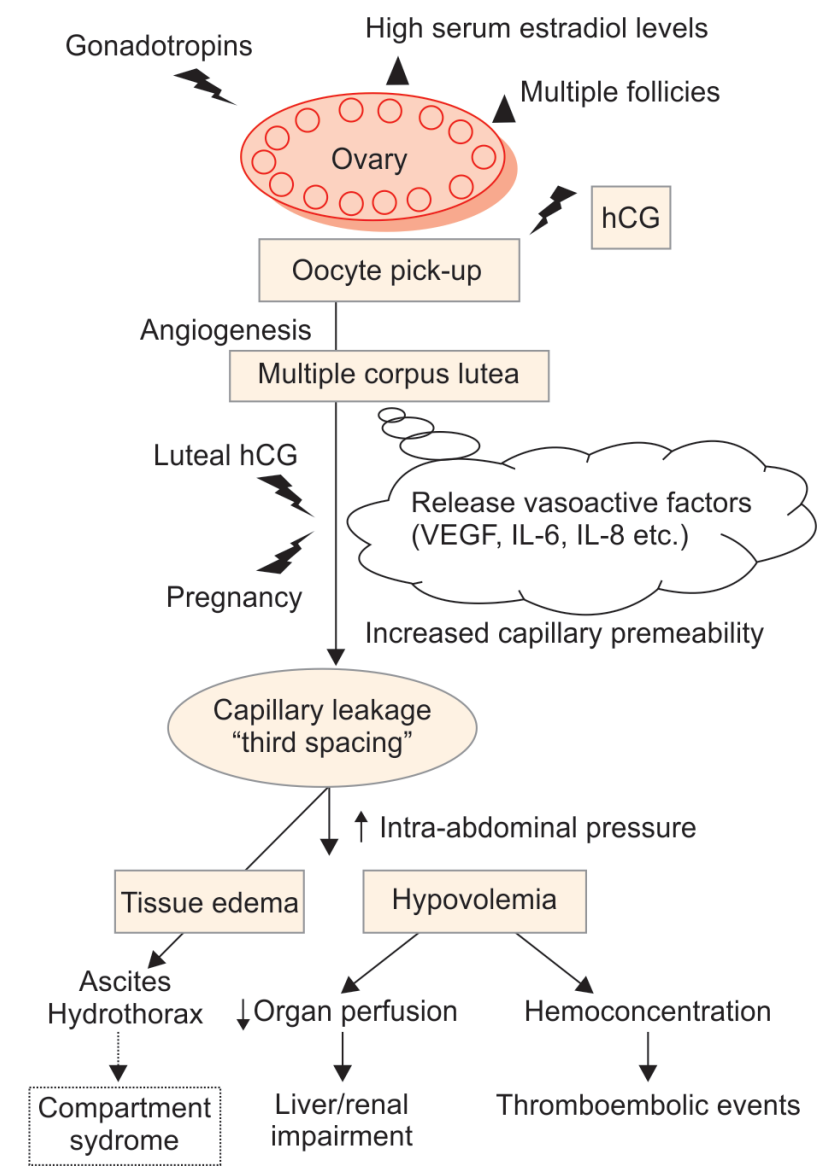

Fig. 1: Pathogenesis of ovarian hyperstimulation syndrome

binds to cell membranes by its receptors. Downstream signaling augments vascular permeability. ${ }^{12}$

Intra-ovarian renin-angiotensin system (RAS) is another mechanism involved in regulating vascular permeability, endothelial proliferation, angiogenesis, and prostaglandin release. Evidence of high renin activity in follicular fluid (FF) proves this. RAS along with increasing VEGF levels synergistically potentiate OHSS with positive correlation with oocyte retrieval rate.

Numerous mediators such as angiotensin II, insulin-like growth factor 1 , and interleukin- 6 are implicated in the process..$^{13}$ Other mechanisms possible for OHSS development are FSH receptor variability as assessed by De Leener et al.

\section{Identifying "At-risk" Woman}

Awareness of risk factors is important for clinicians to predict and preempt occurrence of OHSS to reduce its incidence as a complication of controlled ovarian stimulation.

- Primary risk factors: Young age, low BMI, PCOS, high AMH, and previous history of OHSS. Studies have demonstrated that $\mathrm{AMH}>1.26 \mathrm{ng} / \mathrm{mL}$ is predicted as a risk factor. ${ }^{14}$ Absolute serum estradiol concentrations is a poor predictor. Recent studies have shown that an AFC of more than equal to 24 is estimated to be an important predictor for occurrence of moderate to severe OHSS.

- Secondary Risk Factors: Rapidly rising E2 level (E2 5,000 ng/L and/or $\geq 18$ follicles), $>14$ follicles with a diameter of $11 \mathrm{~mm}$ on trigger day. ${ }^{15,16}$

\begin{tabular}{l} 
Table 1: Classification of ovarian hyperstimulation syndrome (OHSS) \\
\hline Mild OHSS: \\
A. Mild abdominal pain \\
B. Abdominal bloating \\
C. Ovarian diameter $<8 \mathrm{~cm}^{3}$ \\
Moderate OHSS: \\
A. Nausea and/or vomiting \\
B. Moderate abdominal pain \\
C. Ultrasound evidence of ascites \\
D. Ovarian diameter $8-12 \mathrm{~cm}^{3}$ \\
Severe OHSS: \\
A. Clinical ascites with or without hydrothorax \\
B. Oliguria \\
C. Ovarian diameter $>12 \mathrm{~cm}^{3}$ \\
D. Hemoconcentration with hematocrit $>45 \%$ \\
E. Hypoproteinemia \\
Critical OHSS: \\
A. Tense ascites or large hydrothorax \\
B. Oliguria or anuria \\
C. Hematocrit $>55 \%$ \\
D. WBC $>25,000 / \mathrm{mL}$ \\
E. Adult respiratory syndrome \\
F. Thromboembolism
\end{tabular}

\section{Classification and Clinical Features}

The clinical symptoms and signs of OHSS occur in a continuity and to classify them or categorize into stages according to severity is literally a challenge. There are many different classification systems proposed for OHSS. RCOG (2006) have classified OHSS into mild, moderate, severe, and critical subtype (Table 1). Mild OHSS has abdominal bloating and pain. ${ }^{17}$ Severe OHSS has rapid weight gain, clinical ascites, oliguria, hematocrit $>45 \%$, respiratory difficulty, hypoproteinemia, hyperkalemia, and creatinine $>1.2 \mathrm{mg} /$ dL. Critical OHSS is identified with more severe signs with tense ascites and anuria.

Each and every organ system is affected. Pleural effusion, paralytic ileus, and ARDS may occur. ${ }^{18,19}$ Cardiovascular system also deteriorates. Coagulation abnormalities include hemoconcentration, hypercoagulability, and venous thrombosis. ${ }^{20}$ Renal system findings include decreased renal perfusion with subsequent oliguria or renal failure. Severe cases may present with fever; although its etiology is not clear, it may be due to cytokines and prostaglandins released into circulation. ${ }^{21,22}$

\section{Prevention}

\section{"Prevention is the Daughter of Intelligence"}

Prevention strategies for OHSS can be studied as primary and secondary. Primary prevention is based on assessment of a patient's profile and identifying risk factors and working on them. Secondary prevention helps in early diagnosis and intervention. ${ }^{23}$ Deterrence and early detection of OHSS are the most important strategies for the patient's safety. ART centers should tie up with emergency units and hospitals so that appropriate and proper care can be provided. ${ }^{24}$ Young, thin women with polycystic ovaries on ultrasound ( $>12$ antral follicles in each ovary) are at higher risk for OHSS, and thus lower starting 
dose of gonadotropin should be used. ${ }^{25}$ Careful monitoring is prudent and robust monitoring is required to detect early hyperresponse and thus taper the dose.

Women identified with risk factors should have modified treatment according to their history and clinical profile so as to curtail an overexcessive response.

- Aim unifollicular ovulation: In PCOS women, risk of OHSS increases. Goal is unifollicular growth preventing OHSS progression. This can be done by reducing gonadotropin dose by following "step-up" regimen over a "step-down" regimen with 75 IU FSH low dose.

- Avoiding adjunct GnRH agonist (GnRHa) utilization. ${ }^{26}$

- Reducing the gonadotropin duration.

- Utilizing adjuvant metformin therapy.

- Letrozole use to downregulate estrogen production by inhibiting cytochrome $\mathrm{P} 450$ enzyme complex. This promotes increased FSH and central feedback mechanism still remains intact leading to reduced incidence of OHSS during OI though Cochrane review fails to show any added benefit. ${ }^{27}$

\section{Preventive Therapy Modalities during Stimulation 28}

- Cycle cancelation: Cycle cancelation before trigger can prevent OHSS, but the emotional and financial burden it imposes on patients should be considered before the cycle is canceled. This is not a first choice for any clinician.

- Coasting: Coasting involves temporarily stopping gonadotropin administration and postponing the hCG trigger until the estradiol level is lower causing decreased luteinization with lower LH levels. Granulosa cells apoptosis follows. Documented evidence shows that coasting should not exceed more than 3 days.

- Follicular aspiration

- Administration of intravenous albumin and HES: Colloid infusion at time of opu are useful. It binds to vasoactive mediators and prevent OHSS. Albumin has a drawback of anaphylactic reactions and transmission of viral infections and prion disease. ${ }^{29}$ These cannot be overseen. Hydroxyethyl starch (HES) can be used as an alternative to albumin. ${ }^{30}$ The Cochrane review by Youssef et al. noted that there was a statistically significant decrease in severe OHSS (OR 0.12; 95\% Cl 0.04-0.40) with HES use without any effect on pregnancy rates.

- Triggering ovulation with GnRH agonist for final oocyte maturation: Oocyte donor cycles and fertility preservation cycles in which subsequent embryo transfer is not a concern, $\mathrm{GnRH}$ antagonist pituitary suppression protocol is recommended. Here compromised luteal phase is not a concern, as embryos are not being transferred into the patient being stimulated. ${ }^{31}$

- Cryopreservation (eluding endogenous hCG production): Cochrane review has illustrated insufficient evidence to support routine cryopreservation. Segmentation IVF concept given by Paul Devroey for implementation of OHSS-free clinic implies agonist trigger followed by cryopreservation of embryos and transferring single embryo as frozen embryo transfer. But as clinicians, we should be acquainted with the fact that even sequential protocol with freeze all technique does not negate OHSS incidence. Strengths, weaknesses, opportunities, and threats (SWOT) analysis by Blockeel et al. has thrown light on different aspects regarding use of freeze all strategy for prevention of OHSS.

- Dopamine agonist therapy: Recent evidence shows that administration of cabergoline or quinagolide can decrease incidence of OHSS by targeting nonphosphorylation of VEGFR-2. ${ }^{32}$ Recent trials successfully compared use of $0.5 \mathrm{mg} /$ day cabergoline with intravenous albumin. ${ }^{33}$

- Low-dose aspirin therapy.

- In vitro maturation

- Metformin: Cochrane analysis and meta-analysis corroborates its use causing reduction in OHSS incidence (12/216 vs 44/210; OR $0.21,95 \% \mathrm{Cl} 0.11$ to $0.41, \mathrm{P}<0.01)$. Metformin should be started 8 weeks before stimulation and continue till egg retrieval.

- Individualized stimulation protocols: A recent cell culture study reported that $\mathrm{GnRH}$ agonist had direct effect on progesterone secretion by reducing it and negative effect on $\mathrm{LH}$ receptor and FSH receptor mRNA expression.

- Elective single embryo transfer is advised in patients at risk for OHSS. This may decrease the risk of multiple pregnancy, in turn decreasing severe form of OHSS. Progesterone is used for LPS. ${ }^{34}$

Using $\mathrm{GnRH}$ antagonist protocols enable the clinician to use agonist as trigger for final oocyte maturation. GnRH agonist trigger displaces the $\mathrm{GnRH}$ antagonist from its receptor to induce a controlled surge of endogenous LH and FSH. This GnRH agonistinduced LH has a shorter half-life than exogenous hCG, resulting in a less sustained luteotrophic stimulation and hence a lower risk for OHSS especially early OHSS. More aggressive LPS protocol has to be used with agonist trigger to achieve high IVF success rates. ${ }^{35}$

\section{Management}

\section{“They Can't Rescue You if They Don't Know You Need It"}

Diagnosis of OHSS is clinical. There will be preceding history of stimulation in most of cases, but absence of it does not rule out OHSS. Therefore, conditions simulating it should be ruled out. Treatment of the acute phase merely relies on an empirical and symptomatic approach. More adequate methods would require better understanding of the underlying pathophysiological mechanisms to promote an etiological therapeutic approach.

Outpatient management is appropriate for mild, moderate, and few selected severe OHSS cases. Patients have to be properly counseled and informed about oral fluid intake and urine output tabulation. If urine output is less than $1000 \mathrm{~mL}$ in 1 day, then it should be reported to medical facility for prompt action and care. Intravenous hydration should always be initiated with a crystalloid solution to prevent hemoconcentration and provide proper sufficient end-organ perfusion and strict charting to be done (Table 2). If not maintained, then colloid should be given. ${ }^{36}$

Oliguria despite adequate fluid replacement responds to paracentesis in few cases. Multidisciplinary monitoring should be done. Diuretics should not be used without correcting dehydration as it worsens hypovolemia.

Indications for paracentesis include the following: severe abdominal distension, shortness of breath due to ascites, and increased intra-abdominal pressure and failed volume replacement causing oliguria. Paracentesis should be reserved for patients not responding to conservative management because there is a negligible risk of injury to hypervascularized organs. It has been suggested that early drainage of ascites to lower the intra- 


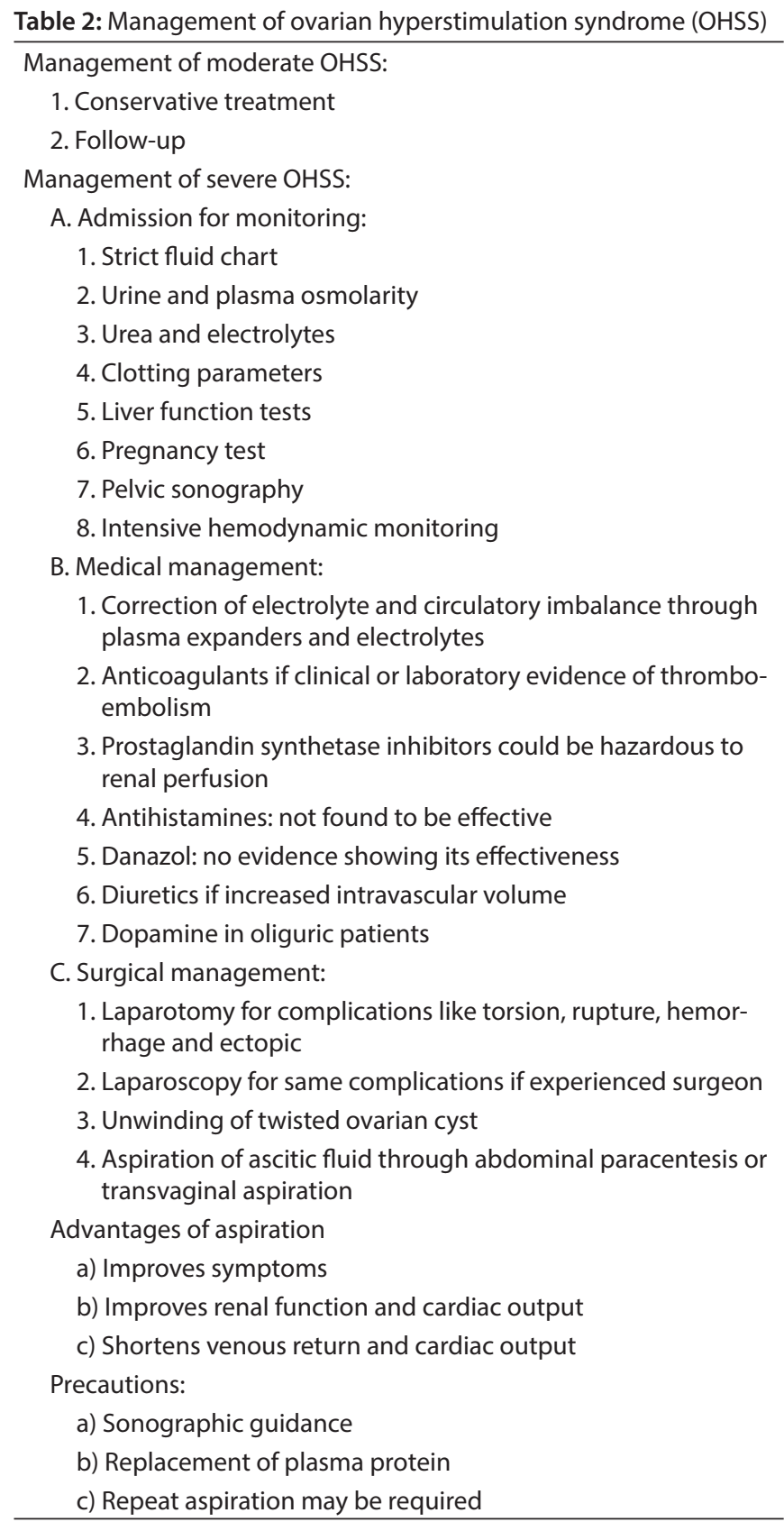

abdominal pressure in patients withholds disease progression and stops worsening of condition. ${ }^{37}$

Women with severe OHSS should receive thromboprophylaxis with LMWH anti-embolism stockings. Individualized duration is suggested. Thrombosis can occur in $0.7-10 \%$ cases of OHSS. Clinical hematologist should look into such cases. Most patients have recovery after thrombosis, although neurological deficits may occur.

C-reactive protein levels may correlate with severity of OHSS. Analgesia and anti-emetics can be used on and off according to symptoms. Avoid NSAIDs and drugs that are contraindicated in pregnancy.

Observational studies suggest that $\mathrm{GnRH}$ antagonist administration may also help in severe early cases although there is insufficient evidence.

\section{Monitoring}

We as clinicians have ethical and medical duty of being vigilant for signs of OHSS and its severity. More frequent assessment is required in cases of severe and critical OHSS. Ovarian hyperstimulation syndrome is self-limiting in majority of women. Monitoring is useful in identifying severity of it and what all measures are required further.

Inpatient monitoring includes body weight, abdominal girth fluid intake/output, blood count, electrolytes, and liver function tests. Depending on the clinical features, arterial blood gases and imaging may be required. Increase in abdominal girth, weight, and hematocrit are signs of worsening OHSS.

Patients with severe and critical OHSS must be provided with invasive hemodynamic monitoring. ${ }^{38}$ Specific complications such as thromboembolism, ARDS, and renal failure require specialized multidisciplinary approach.

Vasopressin-Induced VEGF Secretion Blockade by Vasopressin V1 receptor antagonist "RELCOVAPTAN" has been recently seen to inhibit VEGF by modulating vasoconstriction and vascular smooth muscle proliferation. Rat models have shown this relation of VEGF-A with corpora lutea.

\section{Surgical Management}

This is necessary in extreme cases of complications that arise as a consequence of OHSS. Rarely, pregnancy termination has been reported in progressive thrombosis cases, despite anticoagulation. Intractable OHSS has resulted in bilateral oophorectomy. ${ }^{39}$ Surgery when indicated should be performed by an experienced surgeon. ${ }^{40}$

Compromised obstetric outcome has been reported by many authors. This is due to altered progesterone/estradiol ratios and impaired endometrial receptivity. Some cytokine gene polymorphisms have been associated with increased chances of preeclampsia, miscarriages, and placental insufficiency. Antenatal complications such as hypertension, diabetes, and abruptio placenta are common. Incidence of fetal malformations is not elevated.

Infections are often iatrogenic due to catheterization, pleural puncture, paracentesis, and other surgical procedures. These are nosocomial infections involving bacteria such as Pseudomonas, Proteus, Klebsiella, and Enterobacter. Thus, hospitalization should be minimum and ambulation should be favored.

\section{Counseling}

Couple should be properly counseled about the condition and that management is ultimately supportive only and condition will resolve on its own spontaneously. Consent both verbal and written should be taken before start of any ART procedure. Natural history of OHSS and all other complications should be made understandable to the patient in a language known to her. Couple should be well versed and informed about the admission in the hospital and all its consequences. Meanwhile, reassurance is also necessary.

\section{Conclusion}

OHSS is a rare iatrogenic complication; nevertheless, it may be of severe variety leading to significant morbidity. Ovarian hyperstimulation syndrome is a complication of fertility treatment, which uses pharmacological ovarian stimulation to increase the number of oocytes and therefore embryos available during ART. 
Ovarian hyperstimulation syndrome is associated with significant physical and psychosocial morbidity and mortality. Most cases are self-limiting and require supportive management for resolution to be complete. Women with severe OHSS may need hospital admission to reduce chances of complications. Early detection and care are the key fundamentals in moderate and severe OHSS cases.

Data are not up to date and limited till now causing difficulty to prioritize superiority of one method over other method. Continued research is needed for understanding the pathophysiology of OHSS.

Based on recent data, one can hypothesize that ovarian stimulation protocols should be individualized, and already proven approaches combined together should be the target. These can be use of metformin, GnRH-antagonists, low-dose gonadotropins with Letrozole co-treatment, agonist trigger, and "freeze all" with frozen embryo transfer, dopamine antagonists, and/or GnRH-ant in luteal phase. These might be more beneficial when dealt with all together or in combination. ${ }^{41}$ Outcome and effectiveness of such combined approach is yet to be proven through large-scale randomized controlled trials.

\section{References}

1. Smith V, Osianlis T. Prevention of ovarian hyperstimulation syndrome: a review. Obstet Gynecol Int. 2015;2015:514159.

2. Humaidan P, Quartarolo J, Papanikolaou EG. Preventing ovarian hyperstimulation syndrome: guidance for the clinician. Fertil Steril 2010;94(2):389-400. DOI: 10.1016/j.fertnstert.2010.03.028.

3. Tan BK, Mathur R. Management ofovarian hyperstimulation syndrome. Produced on behalf of the BFS policy and practice committee. Hum Fertil 2013;16(3):151-159. DOI: 10.3109/14647273.2013.788313.

4. Royal College of Obstetricians and Gynaecologists: RCOG Green-top Guideline No. 5.

5. Olivennes F, Howles C, Borini A, et al. Individualizing FSH dose for assisted reproduction using a novel algorithm: the CONSORT study. Reproduct Bio Med Online 2009;18(2):195-204. DOI: 10.1016/S14726483(10)60256-8.

6. Delvinge A, Rozenberg S. Epidemiology and prevention of ovarian hyperstimulation syndrome (OHSS): a review. Hum Reprod Update 2002;8(6):559-577. DOI: 10.1093/humupd/8.6.559.

7. anTilborg TCv, Eijkemans MJC, Lavenetal JSE. The OPTIMIST study: optimisation of cost effectiveness through individualised FSH stimulation dosages for IVF treatment. A randomised controlled trial. BMC Women's Health 2012;12(1):29. DOI: 10.1186/1472-6874-12-29.

8. Evbuomwan IO, Davison JM, Murdoch AP. Coexistent hemo concentration and hypoosmolality during superovulation and in severe ovarian hyperstimulation syndrome: a volume homeostasis paradox. Fertil Steril 2000;74(1):67-72. DOI: 10.1016/S00150282(00)00573-2.

9. Ata B, Tulandi T. Pathophysiology of ovarian hyperstimulation syndrome and strategies for its prevention and treatment. Expert Rev Obstet Gynecol 2009;4(3):299-311. DOI: 10.1586/eog.09.10.

10. Mathur RS, Akande AV, Keay SD, et al. Distinction between early and late ovarian hyperstimulation syndrome. Fertil Steril 2000;73(5):901907. DOI: 10.1016/S0015-0282(00)00492-1.

11. Gomez R, Gonzalez-Izquierdo M, Zimmermann RC, et al. Low-dose dopamine agonist administration blocks vascular endothelial growth factor (VEGF)-mediated vascular hyperpermeability without altering VEGF receptor 2-dependent luteal angiogenesis in a rat ovarian hyperstimulation model. Endocrinology 2006;147(11):5400-5411. DOI: 10.1210/en.2006-0657.

12. Rizk B, Aboulghar M, Smitz J, et al. The role of vascular endothelial growth factor and interleukins in the pathogenesis of severe ovarian hyperstimulation syndrome. Hum Reprod Update 1997;3(3):255-266. DOI: 10.1093/humupd/3.3.255.

13. Enskog A, Henriksson M, Unander M, et al. Prospective study of the clinical and laboratory parameters of patients in whom ovarian hyperstimulation syndrome developed during controlled ovarian hyperstimulation for in vitro fertilization. Fertil Steril 1999;71(5):808814. DOI: 10.1016/S0015-0282(99)00090-4.

14. Gnoth C, Schuring AN, Friol K, et al. Relevance of anti-Mullerian hormone measurement inaroutine IVF program. Hum Reprod 2008;23(6):1359-1365. DOI: 10.1093/humrep/den108.

15. Dewailly D, Andersen CY, Balenetal A. The physiology and clinical utility of anti-Mullerian hormone in women. Hum Reprod Update 2014;20(3):370-385. DOI: 10.1093/humupd/dmt062.

16. Lee T-H, Liu C-H, Huangetal C-C. Serumanti-Müllerian hormone and estradiol levels as predictors of ovarian hyperstimulation syndrome in assisted reproduction technology cycles. Hum Reprod 2008;23(1):160-167. DOI: 10.1093/humrep/dem254.

17. Aboulghar M. Symposium: update on prediction and management of OHSS prevention of OHSS. Reprod Biomed Online 2009;19(1):33-42. DOI: 10.1016/S1472-6483(10)60043-0.

18. Abramov Y, Elchalal U, Schenker JG. Pulmonary mani festations of severe ovarian hyperstimulation syndrome: a multicenter study. Fertil Steril 1999;71(4):645-651. DOI: 10.1016/S0015-0282(98)00528-7.

19. Zosmer A, Katz Z, Lancet M, et al. Adult respiratory distress syndrome complicating ovarian hyperstimulation syndrome. Fertil Steril 1987;47(3):524-526. DOI: 10.1016/S0015-0282(16)59069-4.

20. Kim HC, Kemmann E, Shelden RM, et al. Response of blood coagulation parameters to elevated endogenous 17 beta-estradiol levels induced by human menopausal gonadotropins. Am J Obstet Gynecol 1981;140(7):807-810. DOI: 10.1016/0002-9378(81)90744-4.

21. Kermode AG, Churchyard A, Carroll WM. Stroke complicating severe ovarian hyperstimulation syndrome. Aust N Z J Med 1992;23(2):219220. DOI: 10.1111/j.1445-5994.1993.tb01823.x.

22. Engel T, Jewelewicz R, Dyrenfurth I, et al. Ovarian hyperstimulation syndrome. Report of a case with notes on pathogenesis and treatment. Am J Obstet Gynecol 1972;112(8):1052-1060. DOI: 10.1016/0002-9378(72)90180-9.

23. Navot D, Bergh PA, Laufer N. Ovarian hyperstimulation syndrome in novel reproductive technologies: prevention and treatment. Fertil Steril 1992;58(2):249-261. DOI: 10.1016/S0015-0282(16)55188-7.

24. Mathur R, Evbuomwan I, Jenkins J. Prevention and management of ovarian hyperstimulation syndrome. Curr Obstet Gynaecol 2005;15(2):132-138. DOI: 10.1016/j.curobgyn.2005.01.003.

25. Engmann L, Maconochie N, Sladkevicius $P$, et al. The outcome of in-vitro fertilization treatment in women with sonographic evidence of polycystic ovarian morphology. Hum Reprod 1999;14(1):167-171. DOI: 10.1093/humrep/14.1.167.

26. Coomarasamy A, Afnan M, Cheema $D$, et al. Urinary hMG versus recombinant $\mathrm{FSH}$ for controlled ovarian hyperstimulation following an agonist long down-regulation protocol in IVF or ICSI treatment: a systematic review and meta-analysis. Hum Reprod 2008;23(2):310315. DOI: 10.1093/humrep/dem305.

27. Golan A, Ron-el R, Herman A, et al. Ovarian hyperstimulation syndrome: an update review. Obstet Gynecol Surv 1989;44(6):430440. DOI: 10.1097/00006254-198906000-00004.

28. Mathur R, Kailasam C, Jenkins J. Review of the evidence base of strategies to prevent ovarian hyperstimulation syndrome. Hum Fertil 2007;10(2):75-85. DOI: 10.1080/14647270601111239.

29. Kissler S, Neidhardt B, Siebzehnrubl E, et al. The detrimental role of colloidal volume substitutes in severe ovarian hyperstimulation syndrome: a case report. Eur J Obstet Gynecol Reprod Biol 2001;99(1):131-134. DOI: 10.1016/S0301-2115(01)00364-5.

30. Kirshon B, Doody MC, Cotton DB, et al. Management of ovarian hyperstimulation syndrome with chlorpheniramine maleate, mannitol, and invasive hemodynamic monitoring. Obstet Gynecol 1988;71:485-487.

31. Whelan 3rd JG, Vlahos NF. The ovarian hyperstimulation syndrome. Fertil Steril 2000;73(5):883-896. DOI: 10.1016/S0015-0282(00) 00491-X.

32. Ata B, Seyhan A, Orhaner S, et al. High dose cabergoline in management of ovarian hyperstimulation syndrome. Fertil Steril 2009;92(3):1168.e1-1168.e4. DOI: 10.1016/j.fertnstert.2009.05.021. 
33. Garcia-Velasco JA. How to avoid ovarian hyperstimulation syndrome: a new indication for dopamine agonists. Reprod Biomed Online 2009;18(Suppl. 2):71-75. DOI: 10.1016/S1472-6483(10) 60452-X.

34. Pritts EA, Atwood AK. Luteal phase support in infertility treatment: a meta analysis of the randomized trials. Hum Reprod 2002;17(9): 2287-2299. DOI: 10.1093/humrep/17.9.2287.

35. Aboulghar MA, Mansour RT. Ovarian hyperstimulation syndrome: classifications and critical analysis of preventive measures. Hum Reprodu Update 2003;9(3):275-289. DOI: 10.1093/humupd/ dmg018.

36. Aboulghar $\mathrm{M}$, Evers $\mathrm{JH}, \mathrm{Al}$-Inany $\mathrm{H}$. Intravenous albumin for preventing severe ovarian hyperstimulation syndrome: a cochrane review. Hum Reprod 2002;17(12):3027-3032. DOI: 10.1093/humrep/ 17.12.3027.
37. Memarzadeh MT. A fatal case of ovarian hyperstimulation syndrome with perforated duodenal ulcer. Hum Reprod 2010;25(3):808-809. DOI: 10.1093/humrep/dep434.

38. Braat DD, Schutte JM, Bernardus RE, et al. Maternal death related to IVF in the Netherlands 1984-2008. Hum Reprod 2010;25(7):1782-1786. DOI: 10.1093/humrep/deq080.

39. Al Omari W, Ghazal-Aswad S, Sidky IH, et al. Ovarian salvage in bilaterally complicated severe ovarian hyper stimulation syndrome. Fertil Steril 2011;96(2):e77-e79. DOI: 10.1016/j.fertnstert.2011.06.005.

40. Serour Gl, Aboulghar M, Mansour R, et al. Complications of medically assisted conception in 3,500 cycles. Fertil Steril 1998;70(4):638-642. DOI: 10.1016/S0015-0282(98)00250-7.

41. Hambartsoumian E, Tshzmachyan R. Ovarian hyperstimulation syndrome free IVF clinics. Myth or reality? Int J Gynecol and Reprod Sci 2018;1(1):1-2. 